\title{
Subluxação congênita do cristalino: resultados visuais e posição das lentes intraoculares após a cirurgia
}

\author{
Congenital lens subluxation: visual acuity outcomes and \\ intraocular lens postoperative position
}

\author{
Caroline Arraes ${ }^{1}$ \\ Daniela Endriss ${ }^{2}$ \\ Francisco Lobato ${ }^{3}$ \\ João Arraes $^{4}$ \\ Marcelo Ventura ${ }^{5}$
}

\begin{tabular}{l} 
RESUMO \\
\hline Objetivo: Avaliar os resultados visuais e investigar, através da biomi- \\
croscopia ultrassônica, o posicionamento das lentes intraoculares e do \\
anel endocapsular em 17 olhos de 10 portadores de subluxação con- \\
gênita do cristalino, submetidos à mesma técnica cirúrgica pelo \\
mesmo cirurgião. Métodos: O estudo foi realizado no Hospital de \\
Olhos de Pernambuco e Fundação Altino Ventura. A técnica cirúrgica \\
consistiu em facoaspiração com implante de anel endocapsular e de \\
lentes intraoculares com amputação de uma das alças. A idade variou \\
entre 7 e 22 anos. Foram coletados dados sobre acuidade visual para \\
longe pré e pós-operatória, tempo de seguimento após a cirurgia e \\
complicações. Os pacientes foram submetidos à biomicroscopia ul- \\
trassônica. Resultados: O tempo de seguimento médio foi de 2,8 anos. \\
Houve melhora da acuidade visual para longe nos 17 (100\%) olhos: \\
12 olhos (70,6\%) apresentaram acuidade visual para longe melhor que \\
20/40; 4 (23,5\%) apresentaram acuidade visual para longe entre 20/40 \\
e 20/100 e 1 (5,9\%) apresentou acuidade visual para longe pior que \\
20/100, porém melhor que acuidade visual para longe pré-operatória. \\
A opacificação da cápsula posterior ocorreu em 10 olhos (58,9\%). Na \\
biomicroscopia ultrassônica observou-se que todas as lentes intraocu- \\
lares estavam parcialmente descentralizadas, contudo sem atingir o \\
bordo pupilar. Em todos os casos observou-se um adequado posicio- \\
namento do anel e um bom suporte capsular. Conclusão: É possível \\
concluir que o tratamento cirúrgico avaliado proporciona uma boa \\
centralização das lentes intraoculares e do anel endocapsular, com \\
melhora da acuidade visual para longe, sendo uma opção viável, \\
eficaz e segura na reabilitação visual dos pacientes com subluxação \\
congênita do cristalino.
\end{tabular}

Descritores: Subluxação do cristalino; Facoemulsificação; Lentes intraoculares; Microscopia acústica; Síndrome de Marfan; Acuidade visual

\section{INTRODUÇ̃̃̃O}

A subluxação do cristalino pode estar associada a doenças hereditárias sistêmicas como a síndrome de Marfan, homocistinúria, síndrome de WeillMarchesani, hiperlisinemia e deficiência de sulfite oxidase ${ }^{(1)}$. A síndrome de Marfan é a doença mais frequentemente associada ao deslocamento do cristalino, sendo ectopia não progressiva observada em $80 \%$ dos indivíduos. A porção inferior da zônula do cristalino é a região mais afetada o que resulta no deslocamento, que é mais frequentemente súpero-temporal ${ }^{(2-3)}$. 
Na última década, com o avanço da técnica de facectomia com a facoemulsificação, da biocompatibilidade e desenho das lentes intraoculares e dos recursos disponíveis como adjuvantes da cirurgia (fixação escleral de LIO e anel expansor capsular), a facectomia em olhos subluxados com implante primário de lentes intraoculares (LIO) passou a ser uma opção viável ${ }^{(4-5)}$. Em 1993, Witschel e Legler sugeriram o uso do anel expansor endocapsular, com o objetivo de estabilizar o saco capsular e permitir uma facectomia segura e o implante de LIO em posição adequa$\mathrm{da}^{(6)}$. Alguns autores em 1998, apresentaram um anel endocapsular que permite a fixação escleral sem violação do saco capsular para pacientes com diálise ou fraquezas severas da zônula, com excelente suporte e centralização durante a cirurgia e durante o acompanhamento pós-operatório ${ }^{(7)}$.

Na Fundação Altino Ventura e Hospital de Olhos de Pernambuco vem sendo desenvolvida, desde 1999, uma nova proposta cirúrgica para a ectopia do cristalino, que consiste no implante de LIO com uma alça amputada, apoiada no anel endocapsular. Tal técnica foi desenvolvida e vem sendo realizada por um dos autores (MV) deste trabalho ${ }^{(8)}$.

Na biomicroscopia ultrassônica (UBM) utiliza-se ultrassom de alta frequência (transdutores de 50 a $100 \mathrm{MHz}$ ) com ótima resolução para o segmento anterior (profundidade de penetração tecidual máxima de 4-5 $\mathrm{mm}$ ) sendo utilizada para elucidações diagnósticas, como alterações palpebrais, conjuntivais, irianas, corneanas, esclerais e do corpo ciliar. Também é bastante útil no estudo das complicações cirúrgicas das diferentes causas como ceratoplastias, cirurgias refrativas, cirurgias antiglaucomatosas, implantes de lentes intraoculares e cirurgias vitreorretinianas ${ }^{(9-10)}$.

O objetivo do presente estudo foi avaliar, após a cirurgia, os resultados visuais e investigar, através da biomicroscopia ultrassônica, o posicionamento das lentes intraoculares e do anel endocapsular, posicionamento e estrutura anatômica do saco capsular em portadores de subluxação congênita do cristalino.

\section{MÉTODOS}

O estudo prospectivo foi realizado em 17 olhos de 10 pacientes portadores de subluxação congênita do cristalino, submetidos à cirurgia na Fundação Altino Ventura e Hospital de Olhos de Pernambuco, pelo mesmo cirurgião. Todos os pacientes foram submetidos à anestesia geral, com exceção de um com 18 anos de idade, que foi realizada anestesia local peribulbar. Realizou-se incisão escleral tunelizada à aproximadamente $1,5 \mathrm{~mm}$ do limbo, capsulorrexe descentrada em direção à subluxação ou às 12 horas, confeccionada com pinça Utrata. Implantou-se o anel expansor dentro do saco capsular (Mediphacos 12-10) com injetor ou manual, de forma que as extremidades deste permaneçam próximas das 12 horas, fora da área de maior fragilidade zonular. O córtex e núcleo cristaliniano foram aspirados, utilizando-se ponteira de irrigação/aspiração.
Utilizou-se LIO rígida de polimetilmetacrilato (PMMA), com 6,5 $\mathrm{mm}$ na parte óptica e 13,5 mm de alça a alça (Mediphacos). Amputou-se uma das alças da LIO com tesoura, deixando um pequeno coto de aproximadamente $1 \mathrm{~mm}$ para apoiar a LIO no anel endocapsular, na posição desejada. Implantou-se no saco capsular, inicialmente a alça amputada e posteriormente o lado da alça íntegra, deslocando a LIO no saco. A LIO é posicionada para que o coto da alça amputada apóie-se no anel endocapsular na área de maior subluxação, próximo às 8 horas, permitindo a descentralização da parte óptica da LIO para o eixo visual. Não foram observadas complicações durante o implante da LIO, como ruptura do saco capsular.

Foram excluídos do estudo dois pacientes menores de quatro anos de idade, por não colaboração com o exame ultrassonográfico e um paciente que veio a falecer antes da realização do exame. A idade dos pacientes variou entre 7 a 22 anos. Oito eram do gênero masculino e dois do feminino. O tempo de seguimento variou entre 8 meses e 5,7 anos, média de 2,8 anos. A opacificação da cápsula posterior esteve presente em 10 dos olhos estudados (58,9\%). Em todos os casos (10 olhos, $100 \%$ ) foi realizada capsulotomia com Nd:Yag laser, sem intercorrências.

Foram coletados dados sobre: data da cirurgia, lateralidade da subluxação do cristalino, complicações e data das mesmas, além da acuidade visual para longe com correção pré e pósoperatória. Foi realizada investigação através da biomicroscopia ultrassônica do posicionamento das lentes intraoculares implantadas, posicionamento e estrutura anatômica do saco capsular destes pacientes. Classificou-se da seguinte forma quanto ao grau de centralização das lentes intraoculares: grau I LIO completamente centralizada; grau II - LIO parcialmente descentralizada não atingindo bordo pupilar; grau III - LIO descentralizada em bordo pupilar; grau IV - LIO descentralizada ultrapassando o bordo pupilar.

Foi utilizado o teste $t$ de Student para amostras pareadas com a finalidade de comparar a média do tempo de seguimento após a cirurgia. Para comparar a distribuição da AVL pré e pósoperatória foi utilizado o teste de homogeneidade marginal. Em todos os testes foi utilizado o nível de significância de 5\%.

Este estudo segue os termos preconizados pelo Conselho Nacional de Saúde para pesquisa em seres humanos e foi aprovado pelo Comitê de Ética em Pesquisa da Fundação Altino Ventura. O termo de consentimento e esclarecimento foi assinado pelos responsáveis destes pacientes.

\section{RESULTADOS}

Os resultados das acuidades visuais para longe com correção além do tempo de seguimento após cirurgia e complicações dos 17 olhos submetidos a tratamento cirúrgico para correção da subluxação congênita do cristalino, estão descritos nas tabelas 1 e 2 .

A média do seguimento após cirurgia dos pacientes que apresentaram opacificação de cápsula posterior (46,3 $\pm 15,5$ 


\begin{tabular}{|c|c|c|c|c|}
\hline $\begin{array}{l}\text { Pacientes } \\
\text { (olhos) }\end{array}$ & $\begin{array}{l}\text { AVL c/c } \\
\text { Pré-op }\end{array}$ & $\begin{array}{l}\text { AVL c/c } \\
\text { Pós-op }\end{array}$ & $\begin{array}{l}\text { Seguimento } \\
\text { Pós-op* }\end{array}$ & OCP \\
\hline 1 & $20 / 300$ & $20 / 80$ & 36 & + \\
\hline 2 & $20 / 80$ & $20 / 40$ & 29 & + \\
\hline 3 & $20 / 130$ & $20 / 100$ & 45 & + \\
\hline 4 & $20 / 400$ & $20 / 150$ & 46 & + \\
\hline 5 & $20 / 200$ & $20 / 70$ & 45 & + \\
\hline 6 & $20 / 200$ & $20 / 40$ & 53 & - \\
\hline 7 & $20 / 150$ & $20 / 20$ & 55 & + \\
\hline 8 & $20 / 100$ & $20 / 20$ & 53 & + \\
\hline 9 & $20 / 150$ & $20 / 70$ & 8 & - \\
\hline 10 & $20 / 150$ & $20 / 25$ & 19 & + \\
\hline 11 & $20 / 100$ & $20 / 25$ & 17 & - \\
\hline 12 & $20 / 200$ & $20 / 25$ & 8 & - \\
\hline 13 & $20 / 50$ & $20 / 25$ & 10 & - \\
\hline 14 & $20 / 60$ & $20 / 40$ & 10 & - \\
\hline 15 & $20 / 60$ & $20 / 40$ & 12 & - \\
\hline 16 & $20 / 100$ & $20 / 25$ & 68 & + \\
\hline 17 & $20 / 100$ & $20 / 25$ & 67 & + \\
\hline
\end{tabular}

\begin{tabular}{|c|c|c|c|c|}
\hline \multirow[t]{2}{*}{ AVL c/c } & \multicolumn{2}{|c|}{ Pré-operatória } & \multicolumn{2}{|c|}{ Pós-operatória } \\
\hline & $n$ & $\%$ & $\mathbf{n}$ & $\%$ \\
\hline I. $0,5-1$ (20/20-20/40) & 0 & - & 12 & 70,5 \\
\hline II. $0,2-0,5(20 / 40-20 / 100)$ & 4 & 23,5 & 4 & 23,5 \\
\hline III. $<0,2$ (pior que 20/100) & 13 & 76,5 & $1^{*}$ & 6,0 \\
\hline
\end{tabular}

meses) foi significantemente maior do que a dos pacientes que não apresentaram esta complicação (16,9 $\pm 16,2$ meses); $(\mathrm{t}=3,78-\mathrm{p}=0,002)$.

Através da UBM observou-se que todas as alças cirurgicamente amputadas foram localizadas nos 17 olhos, assim como seu posicionamento foi determinado. Foi encontrado grau II com relação à centralização das lentes, ou seja, nos 17 olhos $(100 \%)$ havia discreta descentralização (não atingindo o bordo pupilar), verificando-se em todos os casos um adequado posicionamento do anel e um bom suporte capsular.

\section{DISCUSSÃO}

Vários tratamentos, ao longo dos anos, têm sido sugeridos para pacientes com subluxação cristaliniana. Desde a simples observação, para casos assintomáticos ou com alterações ópticas corrigíveis com óculos ou lentes de contato, até procedimentos mais invasivos, como lensectomia via pars-plana sem implante de LIO ou zonulólise enzimática da porção luxada da cápsula com intenção de deslocar o cristalino do eixo visual, estabelecendo uma condição de pseudofacia $^{(11)}$.

Na última década a facectomia com implante de LIO passou a ser uma opção viável. Na ectopia do cristalino a perda significante de suporte zonular dificulta o procedimento cirúrgico e o implante da LIO. O anel intracapsular ajuda a manter o contorno circular do saco capsular, evitando a descentração da LIO, exceto em alguns casos com extensa fragilidade zonular, em que muitas vezes não é possível a adequada centralização do saco capsular e da LIO. Através da técnica cirúrgica utilizada de facoaspiração, implante de anel endocapsular e implante de LIO com amputação de uma das alças, a LIO é manipulada para que o coto da alça amputada apóie-se sobre o anel endocapsular, permitindo o deslocamento da parte óptica da LIO para o eixo visual. Sugere-se que com esta técnica é possível manter a integridade evitando a tensão no saco capsular ${ }^{(8)}$.

No presente estudo foi observado que a acuidade visual pós-operatória foi melhor nos 17 olhos estudados (100\%). Não houve complicação intraoperatória. A complicação encontrada inerente ao ato cirúrgico foi a opacidade de cápsula posterior (OCP), presente em 10 olhos (58,9\%). A OCP ocorre frequentemente após facectomia em crianças, estando mais relacionada ao manejo intraoperatório da cápsula posterior, do que ao tipo de LIO utilizada, segundo alguns autores ${ }^{(8)}$.

Através da UBM foi possível avaliar o grau de centralização das LIOs implantadas verificando-se que nos 17 olhos (100\%) havia uma discreta descentralização da LIO, porém sem ultrapassar o bordo pupilar, o que permite uma boa acuidade visual mesmo em situações de midríase. Foram avaliados também através da UBM, o anel endocapsular e o saco capsular, sendo observado em todos os casos um adequado posicionamento do anel e um bom suporte capsular.

\section{CONCLUSÃO}

Os resultados obtidos sugerem que o implante de LIO com uma alça amputada proporciona uma boa centralização da LIO e do anel endocapsular, como demonstrado pela UBM, com melhora da AVL, sendo uma opção viável, eficaz e segura na reabilitação visual dos pacientes com subluxação congênita do cristalino. Todavia, as indicações específicas, a segurança em longo prazo e eficácia devem ser melhor avaliadas através de estudo com amostras maiores e com maior tempo de seguimento.

\section{ABSTRACT}

Purpose: To evaluate the visual acuity outcomes and to investigate the intraocular lens (IOL) and endocapsular ring positions with ultrasound biomicroscopy in 17 eyes of 10 patients with congenital lens subluxation who underwent the same surgical technique, by the same surgeon. Methods: The study was performed in the "Hospital de Olhos de Pernam- 
buco" and "Fundação Altino Ventura". The surgical technique consisted of phacoaspiration with implant of endocapsular ring and intraocular lens with one loop haptic amputated. The age varied from 7 to 22 years. Data on visual acuity (VA) before and after surgery, surgery follow-up period, and complications were analyzed. All patients underwent ultrasound biomicroscopy. Results: The mean follow-up period was 2.8 years. There was a VA improvement in 17 (100\%) eyes: in 12 eyes $(70.6 \%)$ the visual acuity was better than 20/40; 4 (23.5\%) ranged from 20/40 to 20/100, and $1(5.9 \%)$ had visual acuity worse than 20/100, however better than the preoperative visual acuity. The posterior capsular opacification occurred in 10 eyes (58.9\%). Ultrasound biomicroscopy showed that all IOL were partially decentralized, however without surpassing the pupil border limit. Endocapsular ring position was correct and there was a good capsular support in all cases. Conclusions: The evaluated surgical treatment provided good intraocular lens and endocapsular ring position, with VA improvement Thus, this technique is a viable, effective and safe option for the visual rehabilitation of patients with congenital lens subluxation.

Keywords: Lens subluxation; Phacoemulsification; Lens, intraocular; Microscopy, acoustic; Marfan syndrome; Visual acuity

\section{REFERÊNCIAS}

1. Anteby I, Isaac M, BenEzra D. Hereditary subluxated lenses: visual performances and long-term follow-up after surgery. Ophthalmology. 2003;110(7):1344-8.

2. Oliveira DF. Anomalias congênitas do cristalino. In: Arieta CEL. Cristalino e catarata. Rio de Janeiro: Cultura Médica; 2002. p.31-47.

3. Halpert M, BenEzra D. Surgery of the hereditary subluxated lens in children. Ophthalmology. 1996;103(4):681-6.

4. Lam DS, Ng JS, Fan DS, Chua JK. Leung AT, Tham CC. Short-term results of scleral intraocular lens fixation in children. J Cataract Refract Surg. 1998; 24(11):1474-9.

5. Cionni RJ, Osher RH. Endocapsular ring approach to the subluxated cataractous lens. J Cataract Refract Surg. 1995;21(3):245-9. Comment in: J Cataract Refract Surg. 2001;27(11):1710-1.

6. Legler U, Witschel B, Lim S. The capsular ring: a new device for complicated cataract surgery. In: $3^{\text {rd }}$ American International Congress on Cataract, IOL and Refractive Surgery: Seattle; 1993.

7. Cionni RJ, Osher RH. Management of profound zonular dialysis or weakness with a new endocapsular ring designed for escleral fixation. J Cataract Refract Surg. 1998;24(10):1299-306.

8. Ventura M, Endriss D. Intraocular lens implantation with one loop haptic amputed for congenital subluxation lens surgical treatment. In: Garg A, Prost ME, Azad R, Crouch ER, Mehta KR, Bovet JJ, et al. Surgical and medical management of pediatric ophthalmology. New Delhi: Jaypee Brothers- Medical Publishers; 2007. v.1, p.248-54.

9. Lobato FT, Sena MF, Stillitano IG, Ribeiro MP, Sampaio I, Arraes T. Posição da lente intraocular de câmara posterior após fixação transescleral: análise pela biomicroscopia ultrassônica. An Fac Med Univ Fed Pernamb. 2002;47(2):135-9.

10. Cronemberger S, Marigo FA, Calixto N. Biomicroscopia ultrassônica. In: Yamame R. editor. Semiologia ocular. Rio de Janeiro: Cultura Médica; 2001. p.367-85.

11. Kanski JJ. Ectopia lentis. In: Kanski JJ. Clinical ophthalmology: a systematic approach. $3^{\text {rd }}$ ed. London: Butterwoorth-Heinemann; 1994. p.307-9. 